\title{
Paper-based electroanalytical devices for accessible diagnostic testing
}

\section{Citation}

Maxwell, E. Jane, Aaron D. Mazzeo, and George M. Whitesides. 2013. “Paper-Based Electroanalytical Devices for Accessible Diagnostic Testing." MRS Bulletin 38 (04) (April): 309314.

\section{Published Version}

doi:10.1557/mrs.2013.56

\section{Permanent link}

http://nrs.harvard.edu/urn-3:HUL.InstRepos:12388523

\section{Terms of Use}

This article was downloaded from Harvard University's DASH repository, and is made available under the terms and conditions applicable to Open Access Policy Articles, as set forth at http:// nrs.harvard.edu/urn-3:HUL.InstRepos:dash.current.terms-of-use\#OAP

\section{Share Your Story}

The Harvard community has made this article openly available.

Please share how this access benefits you. Submit a story.

\section{Accessibility}




\title{
Paper-Based Electroanalytical Devices for Accessible Diagnostic Testing
}

\author{
E. Jane Maxwell ${ }^{\mathrm{a}}$, Aaron D. Mazzeo ${ }^{\mathrm{a}}$, and George M. Whitesides ${ }^{\mathrm{a}, \mathrm{b} *}$ \\ ${ }^{a}$ Department of Chemistry and Chemical Biology, Harvard University \\ 12 Oxford St. Cambridge, MA 02138 \\ ${ }^{b}$ Wyss Institute of Biologically Inspired Engineering, 60 Oxford St. Cambridge, MA 02138 \\ *Towhom correspondence may be addressed: gwhitesides@gmwgroup.harvard.edu
}

\begin{abstract}
Microfluidic paper-based analytical devices ( $\mu$ PADs) use the passive capillary-driven flow of aqueous solutions through patterned paper channels to transport a sample fluid into distinct detection zones that contain the reagents for a chemical assay. These devices are simple, affordable, portable, and disposable; they are, thus, well suited for diagnostic applications in resource-limited environments. Adding screen-printed electrodes to the detection zones of a $\mu \mathrm{PAD}$ yields a device capable of performing electrochemical assays (an E $\mu \mathrm{PAD}$ ).

Electrochemical detection has the advantage over colorimetric detection that it is not affected by interferences from the color of the sample, and can be quantified with simple electronics. The

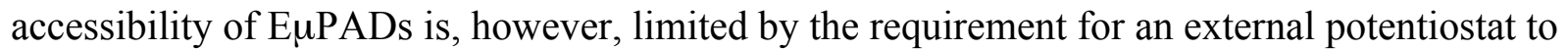
power and interpret the electrochemical measurement. New developments in paper-based electronics may help loosen some of this requirement. This review discusses the current

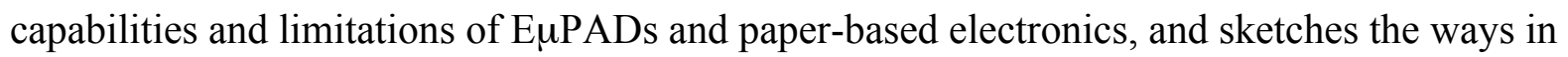
which these technologies can be combined to provide new devices for diagnostic testing.
\end{abstract}

Keywords: micro-paper-based analytical devices, diagnostics, electrochemical sensing, bioanalysis, electroanalytical devices, wax printing, developing world, inexpensive 


\section{The need for accessible diagnostic devices}

Diagnosis is a critical part of the management of disease. Although diagnostic tests, by themselves, contribute a relatively small amount to the total costs of healthcare, the results of diagnosis determine the subsequent treatment and its costs. In the developed world, diagnostic testing is commonly carried out using dedicated instruments in well-equipped central laboratories. The procedure-based reimbursement system encourages the development of sophisticated, and expensive, tests. This type of laboratory-based technology is inappropriate, however, for many applications, especially in resource-limited settings. Affordability, portability, ease-of-use, and the ability to operate with little or no supporting equipment are particularly important for tests used in the developing world, in public health, at the point-ofcare, in drug testing and monitoring, and in agriculture (veterinary, food, environmental testing).

The potential of paper as a substrate for colorimetric diagnostic testing has already been

demonstrated, ${ }^{1-4}$ but there remains a substantial opportunity for paper-based devices that can replicate other commonly used modes of detection - particularly electrochemical detection while retaining their low cost and simplicity. The realization of this opportunity depends on our ability to build electrochemical and electronic capabilities on paper substrates.

\section{Paper-based approaches to bioanalysis}

As a matrix for chemical and biochemical analysis relevant to diagnosis, paper offers five properties that contribute to meeting these requirements: (i) the capillary wicking of aqueous solutions along the cellulose fibers of the paper matrix provides a means of transporting fluids that does not require external energy; (ii) the porosity and biocompatibility of paper allow reagents to be stored or immobilized in the paper device; (iii) the light weight and flexibility of 
paper simplify the logistics of transport and storage; (iv) the patterning and processing of paper can use established methods to form microfluidic channels in paper; (v) the low cost and combustibility of paper make it suitable for disposable, safe, single-use tests.

Paper-based systems are, of course, already extensively used. Test strips for urinalysis use an array of paper test pads, supported on a plastic strip, to perform up to eleven colorimetric assays in urine (glucose, protein, ketones, $\mathrm{pH}$, nitrite, bilirubin, urobilinogen, leukocyte esterase, specific gravity, ascorbic acid, and blood). ${ }^{5}$ Immunochromatographic, "lateral-flow" assays move a sample (and analyte) between different zones on a linear nitrocellulose strip by passive wicking; the zones perform the different steps of an immunoassay (capture of antigen, addition of a labeled antibody for detection, washing) to produce a colored line in the presence of the analyte of interest. ${ }^{6}$

\section{Micro-paper-based analytical devices ( $\mu$ PADs)}

In 2007, we described a new design for a microfluidic device made from paper (a micropaper-based analytical device, or $\mu \mathrm{PAD})$. These devices use hydrophobic barriers patterned on hydrophilic paper by photolithography, ${ }^{7}$ by printing with $\mathrm{PDMS}^{8}$ or dissolved polystyrene, ${ }^{9}$ or by solid wax applied by a commercial printer ${ }^{10,11}$ to create microfluidic channels that guide the transport of fluids into discreet test zones. It is also possible to extend the topology of the microfluidic channels into the third dimension by stacking ${ }^{12}$ or folding ${ }^{13,14}$ sheets of paper to join multiple layers. As in the case of urinalysis test strips, the test zones of a $\mu \mathrm{PAD}$ contain the reagents (enzymes, indicator dyes, buffers) required to generate a change in color in the presence of the analyte of interest. The results can be read by eye, or imaged photographically and analyzed by computer. 


\section{Advantages and limitations of colorimetric $\mu P A D s$}

The portability, affordability, and simplicity of colorimetric $\mu$ PADs make them attractive for use in remote areas, but the interpretation of the results may require expertise or image analysis that is not available at the point of care. Telemedicine offers one potential strategy with which to approach this problem. In this strategy, a cell-phone camera at the point of care captures a photograph of the colorimetric result and transmits the image, via the network used for telephonic communications, to a central location where trained personnel (or a computer) analyze the image and transmit the results back to the user by text message. ${ }^{15}$ Even with the addition of telemedicine, however, colorimetric $\mu$ PADs are not appropriate in every situation. Interferences from the color of the sample, inconsistencies in lighting, or the presence of particulate contaminants may confuse the interpretation of the colorimetric result. For example, colorimetric assays in blood are complicated by the presence of (red) hemoglobin from whole or lysed red blood cells.

\section{Electrochemical readout as an alternative to colorimetry/fluorimetry}

Electrochemical analysis is a useful alternative (or complement) to colorimetric analysis for quantifying the results of a diagnostic test, since the measured current or voltage generated by the electrochemical device is insensitive to color contamination, and can be transformed into a numeric output by inexpensive electronics. Electrochemical analysis can, in principle, be used either independently of or in combination with colorimetric analysis.

There are several examples of successful electroanalytical systems already in large-scale use in point-of-care or clinical laboratory settings in the developed world. ${ }^{16}$ These include personal glucose meters (which are widely used in the management of diabetes), the iSTAT 
Handheld Blood Analyzer (which is used in hospitals to measure electrolytes and other biomarkers in blood), and the ORIGEN Analyzer (a clinical instrument for performing quantitative immunoassays based on electro-chemiluminescence).

\section{Electrochemical capabilities of E $\mu$ PADs}

Adding paper-based electrodes to a $\mu \mathrm{PAD}$ yields a paper-based device capable of performing electrochemical measurements (an E $\mathrm{PAD}) .{ }^{17,18}$ Carbon-based materials (graphite, graphene, or carbon nanotubes), gold, and platinum are appropriate choices for the working and counter electrodes because they are electrochemically inert over a wide range of working potentials. Electrodes constructed from $\mathrm{Ag} / \mathrm{AgCl}$ paste are included in electrochemical measurements that require a reference electrode.

Electrochemical measurements made in paper differ from those performed in free solution due to the presence of the cellulose matrix, which largely blocks convection (in stationary fluids), acts as a barrier to diffusion (by increasing the tortuosity of the diffusion path), occupies some of the volume that would otherwise be occupied by fluid, and blocks a portion of the electrode surface ( $\sim 30 \%$, depending on the type of paper and fabrication method used) ${ }^{22}$ Conversely, the presence of the cellulose matrix is advantageous for hydrodynamic measurements; capillary flow in paper can drive the transport of analytes over the surface of the electrode, resulting in increased sensitivity.

\section{Amperometric analysis of simple metabolites}

Amperometric E $\mu \mathrm{PADs}$ measure the current resulting from the oxidation or reduction of electroactive species at a fixed applied potential. Only a few metabolites with low redox 
potentials, such as ascorbic acid and uric acid, can be measured directly by this technique. More commonly, amperometry is coupled with an enzymatic assay that consumes the analyte of interest and generates an electroactive product (Equation 1). Many groups have demonstrated sensors for glucose, lactate, ethanol, and cholesterol using this principle. ${ }^{17-22}$

$$
\begin{aligned}
& \text { Glucose }+\mathrm{H}_{2} \mathrm{O}+2 \mathrm{Fe}(\mathrm{CN})_{6}^{3-} \stackrel{\text { Glucose oxidase }}{\longrightarrow} \text { Gluconic acid }+2 \mathrm{H}^{+}+2 \mathrm{Fe}(\mathrm{CN})_{6}^{4-} \\
& 2 \mathrm{Fe}(\mathrm{CN})_{6}^{4-} \stackrel{\text { Oxidizing electrode }}{\longrightarrow} 2 \mathrm{Fe}(\mathrm{CN})_{6}^{3-}+2 \mathrm{e}^{-}
\end{aligned}
$$

\section{Voltammetric analysis of trace metals}

Voltammetric methods for the analysis of metals take advantage of the transition from soluble ions to an insoluble solid that occurs when metals are reduced. The measurement occurs in two distinct stages (Equation 2): during an initial deposition stage, which can last several minutes, a reducing potential applied at the working electrode causes metal ions to deposit onto the electrode surface in the form of a solid metal, alloy, or amalgam; during the subsequent measurement, or "stripping" stage, the potential rapidly scans towards more positive values in a linear or square-wave sweep, and causes the deposited metals to reoxidize. The oxidation is observed as a peak in the current at the characteristic redox potential for each metal.

$$
\begin{array}{ll}
\text { deposition: } & \mathrm{M}_{(\mathrm{aq})}^{n+}+n \mathrm{e}^{-} \stackrel{\text { Reducing electrode, }}{\longrightarrow} \mathrm{M}_{(\mathrm{s})} \\
\text { stripping: } & \mathrm{M}_{(\mathrm{s})} \stackrel{\text { Oxidizing electrode }}{\longrightarrow} \mathrm{M}_{(\mathrm{aq})}^{n+}+n \mathrm{e}^{-}
\end{array}
$$

\section{EMPADs employing biomolecular recognition}

There are examples of electrochemical lateral-flow test strips and E $\mu$ PADs that incorporate antibodies, ${ }^{23-29}$ aptamers,${ }^{14}$ or complementary DNA ${ }^{13}$ to capture or label target 
molecules. The biomolecular recognition changes the electrochemical properties of the system as a result of enzyme-catalyzed production of an electroactive species, ${ }^{24,27-29}$ mellitin-induced rupture of liposomes containing ferrocyanide, ${ }^{26}$ localization of cadmium quantum dots on the working electrode ${ }^{25}$ or charge repulsion of electroactive species at the electrode surface. ${ }^{13}$

\section{Paper-based electronic systems}

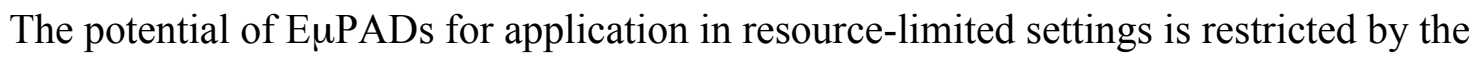
fact that nearly all of the existing devices require an external potentiostat. Although it may not be possible to completely eliminate this requirement, the increasing sophistication of paper-based electronic devices offers hope that some electronic functions may be integrated into the paperbased device itself. Alternatively, the devices needed to carry out more complicated electrochemical measurements using simple, paper-based strips are being developed.

There are a number of recently established techniques for patterning electrodes, ${ }^{18}$ wires,${ }^{30}$ and piezoresistors ${ }^{31}$ on paper, including screen printing ${ }^{32,33}$ direct-writing with a pen/pencil dispensing conductive material, ${ }^{34,35}$ physical deposition of metals, or spraying conductive inks through stencils. ${ }^{30}$ These patterning techniques can also be used to form resistive heating elements on paper. Siegel and coworkers demonstrated a paper-based display that used a resistive heating element to change a layer of thermochromic ink from opaque to transparent and reveal a message selected to summarize the result of a test (Figure 2A). ${ }^{36}$

An alternative strategy for forming circuits on paper involves patterning "disconnects" by removing material from a sheet of metallized paper with laser-based ablation. ${ }^{37}$ This method can be used to create inexpensive paper-based capacitive touch pads capable of replacing the buttons or keypads required for collecting user input (Figure 2B). 
Proof-of-concept studies have demonstrated the potential of fabricating active electronic devices on paper (e.g., ring oscillators ${ }^{38}$ and transistors ${ }^{39-43}$, Figure 2C). Nevertheless, there is still a need to develop manufacturing processes and reduce the cost of fabrication if paper-based transistors are to price comparably with silicon-based electronic components: a standard operational amplifier containing 20 transistors costs $\sim$ US $\$ 0.20$, or $\$ 0.01$ per transistor; ${ }^{44}$ microprocessors containing more than one billion transistors cost less than $\$ 300$, or $<\$ 0.000001$ per transistor. While paper-based microprocessors are not practical at this point, patterning tens or hundreds of transistors on disposable substrates might enable simple signal processing or amplification that would facilitate electrochemical measurements.

Recent academic demonstrations have shown the storage of energy with paper-based supercapacitors $^{45}$ and batteries ${ }^{46-48}$, and the generation of power by solar cells ${ }^{49}$ (Figure 2D). The integration of these elements into E $\mu$ PADs could, in the future, eliminate the need for external power sources.

\section{Combining paper-based electronics and bioanalysis}

One of the advantages of using electrodes patterned on paper is that the analyte only makes contact with a small, low-cost, disposable strip. When external electronics are used to control and analyze electric potentials and currents, they are designed to make no contact with analyte, and thus do not require sterilization or cleaning between uses. The disposable strips only require passive elements that can also have chemically functionalized conductive regions. Disposable electrodes are also preferable to reusable electrodes in a point-of-care setting, because they eliminate the need for reconditioning of the electrode surface. 
Glucometers are an excellent example of a successful electrochemical diagnostic device that combines disposable test strips with a portable electrochemical reader. ${ }^{50}$ They are affordable, easy to use, highly engineered, and globally available; their availability will also increase as the incidence of diabetes and similar metabolic syndromes continues to rise. Based on the success of the glucometer, early applications of EuPADs may follow a similar model.

As an alternative to glucometers, there is a growing interest in adapting "smart" mobile phones (smartphones), which combine capabilities for telecommunications, photography, and computing, to act as readers for point-of-care diagnosis. ${ }^{51,52}$ Most current examples use the built-in camera to capture and process the result of a colorimetric test, but new accessory devices for smartphones, like the iMultimeter, ${ }^{53}$ can be used to measure electrical signals as potentials. With the addition of simple electronic components such as capacitors, switches, and resistors, these devices could act as readers for a variety of electrochemical measurements. ${ }^{14}$ Although smartphones obviously facilitate the communication of results by telemedicine, they are expensive, and the advanced networks required for data transmission are not yet available in many resource-limited environments.

In the future, paper-based devices for performing electrochemical measurements may be completely self-contained and disposable. One existing example, by Liu and coworkers, used a paper-based fluidic battery to power the amperometric oxidation of glucose or hydrogen peroxide, and a spot of electrochromic dye (Prussian blue) that changed color in response to the amperometric current; ${ }^{22}$ the results could be read by eye, as in a colorimetric assay (based on the appearance or disappearance of the Prussian blue spot), and the device required no external electronics. 


\section{Outlook}

With ingenuity, there is the possibility of developing analogs of many electrochemical and electrical systems now used in developed-world medicine that could meet the requirements of low-cost systems. For example, Novell et al. demonstrated paper-based electrodes that incorporated ion-selective membranes for the measurement of $\left[\mathrm{K}^{+}\right],\left[\mathrm{NH}_{3}{ }^{+}\right]$and $\mathrm{pH}$. An E $\mu \mathrm{PAD}$ based on the same principle could be used to quantify serum electrolytes and provide a less expensive and more portable alternative to the iSTAT Handheld Blood Analyzer.

Electrochemiluminescence is a powerful detection technique for immunoassays because the timing of the chemiluminescent emission is controlled by the electrochemical system. This advantage is important in point-of-care settings, because medical personnel juggling multiple patients may be unable to return to interpret the results of a test with precise timing. Paper-based electrochemiluminescent devices have already been demonstrated for the detection of immunoassays. $^{54,55}$

Diagnostic techniques based on the amplification and detection of nucleic acids offer excellent sensitivity and the ability to identify specific strains or mutations in the pathogen of interest. These techniques are particularly challenging to implement in resource-limited settings because the amplification processes require elevated temperatures or thermal cycling. ${ }^{56}$ Paperbased resistive heating elements could be printed directly onto a $\mu$ PAD to permit on-device amplification. Developed methods for patterning electrodes in paper-based electronics could also be used in the measurements of body temperature, pulse, and blood pressure. 


\section{Conclusion}

EuPADs have already been adapted for a wide variety of different types of measurements and analytes. Translating these advances into real point-of-care devices will, however, require a range of new or improved features.

In most bioassays, the characteristic of the assay that ultimately determines performance is the biology (the specificity/binding constant/kinetics of the antibodies, enzymes, etc.), not the electronics. New amplification strategies that increase the sensitivity of EuPADs and reduce the time required for analysis will permit the application of the techniques developed thus far to a wider range of analytes. Amplification strategies that remove the time-dependence of the measured signal, or the dependence on environmental factors, are particularly valuable in pointof-care settings.

The requirement for external equipment to power the electrochemical measurement and detect and process the resulting signal should drive innovations in handheld readers. Despite the advantages of glucometers (cost, availability), they are limited by their design in the types of measurements that they can perform. The development of more versatile portable electrochemical readers that are inexpensive and simple will be an important step for making EuPADs broadly useful. Paper-based electrical devices such as circuit boards, thermochromic displays, buttons, and resistors can perform some of the simpler electronic functions required by EuPADs; the methods used in the fabrication of other, more complex components, including paper-based energy storage devices, transistors, and other active elements on paper, require further development.

Integration of bioanalysis with telemedicine is a core theme for the future of public health and point-of-care medicine. Electrochemical systems seem particularly well suited for 
integration with cell-phone-based systems, since the disposable biosensor (paper) is light, inexpensive, and easy to use, and the output can be an electrical signal.

\section{Acknowledgements}

This work was funded by the Bill \& Melinda Gates Foundation under award number 51308, the N/MEMS S\&T Fundamentals MF3 Center (DARPA), and a postdoctoral fellowship from the Natural Sciences and Engineering Research Council of Canada (E.J.M.). 


\section{Figure Captions}

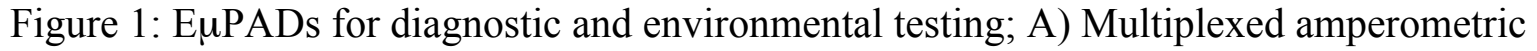
device for the analysis of glucose, lactate, and uric acid ${ }^{18}$; B) Square-wave voltammetry device for the determination of lead in drinking water ${ }^{17}$; C) Amperometric E $\mu$ PAD integrated with a commercial glucometer $^{20}$; D) Adenosine-sensing E $\mu$ PAD with aptamer-based recognition and a potentiometric concentration cell. ${ }^{14}$

Figure 2: Paper-based electronic devices; A) Thermochromic display, i) resistive heating elements, ii) no current applied to resistors, iii) and iv) activation of left and right resistors for the display of different test results; B) Capacitive touch pads fabricated by "disconnection" on metallized paper $^{37}$; C) SEM image of interdigitated electrodes of a paper-based transistor ${ }^{42}$; D) Photovoltaic circuits ${ }^{49}$; E) Piezoresistive paper cantilever for mechanical sensing. ${ }^{31}$ 
Figure 1

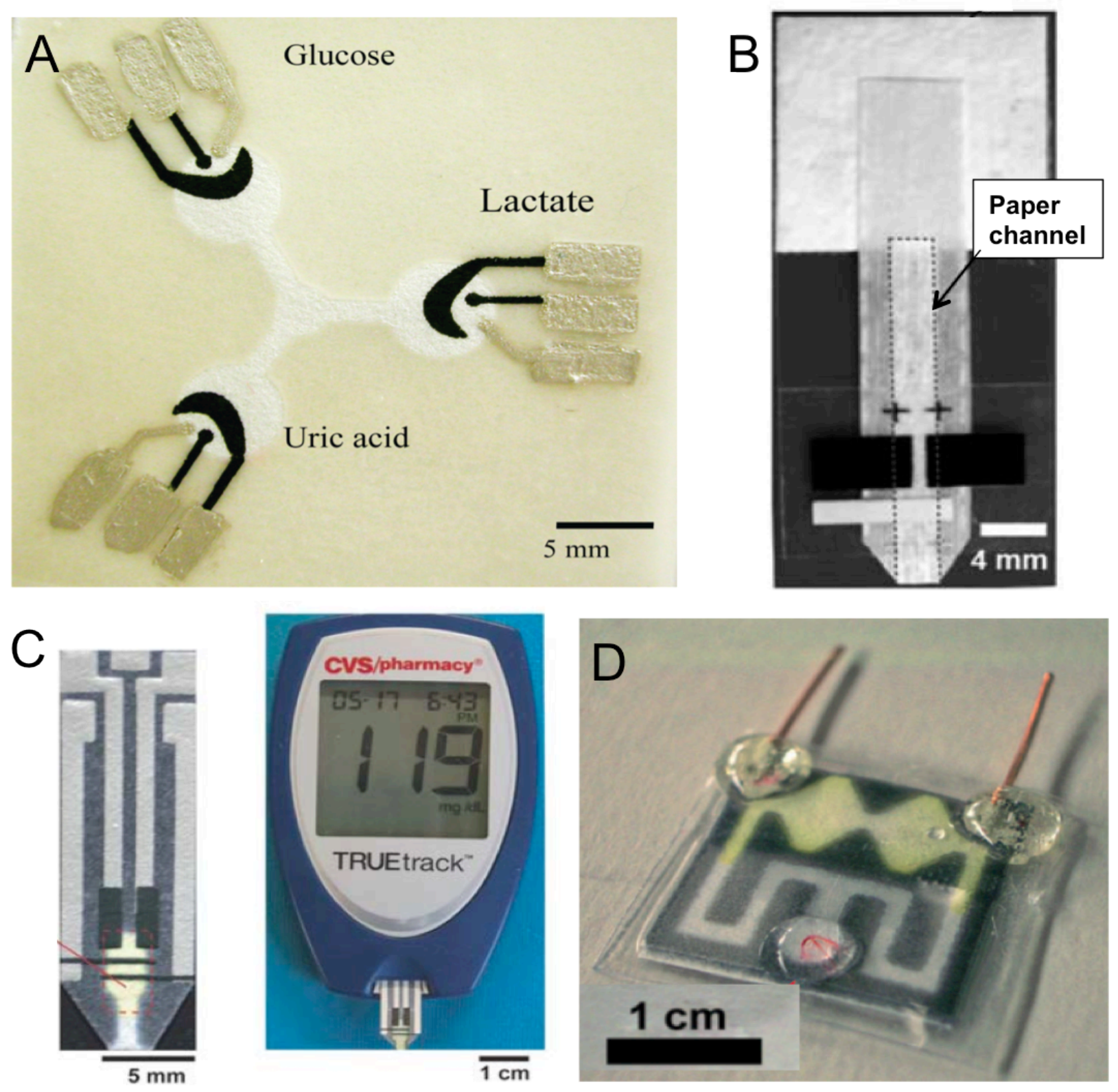


Figure 2
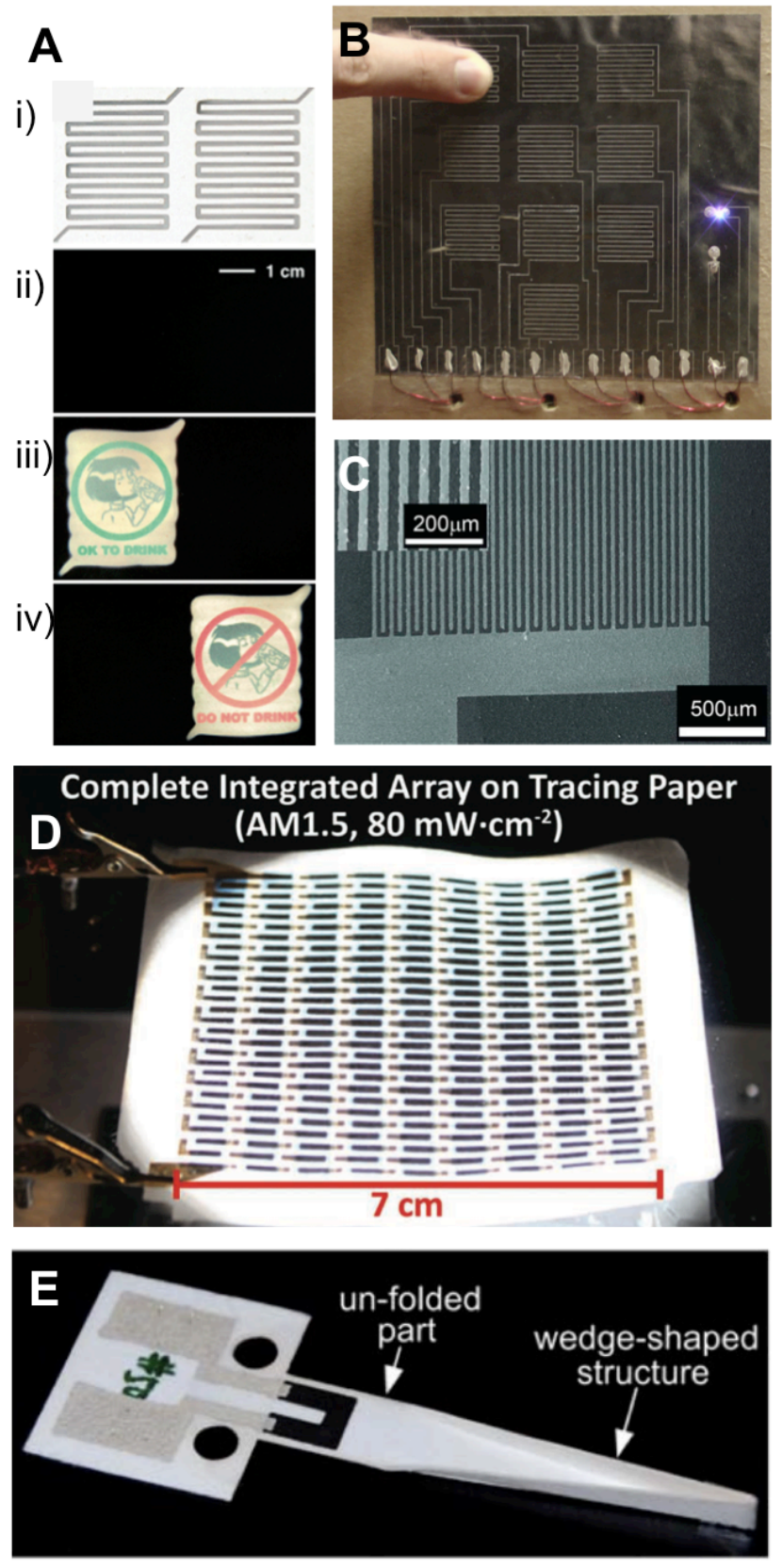


\section{References}

1. $\quad$ A.W. Martinez, S.T. Phillips, G.M. Whitesides, E. Carrilho, Anal. Chem., 823 (2009)

2. E. Fu, T. Liang, P. Spicar-Mihalic, J. Houghtaling, S. Ramachandran, P. Yager, Anal. Chem., 84 4574 (2012)

3. M.S. Khan, G. Thouas, W. Shen, G. Whyte, G. Garnier, Anal. Chem., 824158 (2010)

4. W. Dungchai, O. Chailapakul, C.S. Henry, Anal. Chim. Acta, 674227 (2010)

5. Urinalysis: the Chemical Examination, http://labtestsonline.org/understanding/analytes/urinalysis/ui-exams/start/1, Accessed October 3, 2012.

6. B. O'Farrell, in Lateral Flow Immunoassay, eds. R. Wong, H. Tse, (Humana Press, New York, 2009)

7. A. Martinez, S. Phillips, M. Butte, G. Whitesides, Angew. Chem. Int. Ed., 461318 (2007)

8. D.A. Bruzewicz, M. Reches, G.M. Whitesides, Anal. Chem., 803387 (2008)

9. J. Olkkonen, K. Lehtinen, T. Erho, Anal. Chem., 8210246 (2010)

10. Y. Lu, W. Shi, L. Jiang, J. Qin, B. Lin, Electrophoresis, 301497 (2009)

11. E. Carrilho, A.W. Martinez, G.M. Whitesides, Anal. Chem., 817091 (2009)

12. A.W. Martinez, S.T. Phillips, G.M. Whitesides, PNAS, 10519606 (2008)

13. J. Lu, S. Ge, L. Ge, M. Yan, J. Yu, Electrochim. Acta (2012)

14. H. Liu, Y. Xiang, Y. Lu, R.M. Crooks, Angew. Chem., 1247031 (2012)

15. A.W. Martinez, S.T. Phillips, E. Carrilho, S.W. Thomas, H. Sindi, G.M. Whitesides, Anal. Chem., 803699 (2008)

16. P. D'Orazio, Clin. Chim. Acta, 33441 (2003)

17. Z. Nie, C.A. Nijhuis, J. Gong, X. Chen, A. Kumachev, A.W. Martinez, M. Narovlyansky, G.M. Whitesides, Lab Chip, 10477 (2010)

18. W. Dungchai, O. Chailapakul, C.S. Henry, Anal. Chem., 815821 (2009)

19. G. Cui, S.J. Kim, S.H. Choi, H. Nam, G.S. Cha, K.-J. Paeng, Anal. Chem., 721925 (2000)

20. Z. Nie, F. Deiss, X. Liu, O. Akbulut, G.M. Whitesides, Lab Chip, 103163 (2010)

21. J. Lankelma, Z. Nie, E. Carrilho, G.M. Whitesides, Anal. Chem., 844147 (2012)

22. H. Liu, R.M. Crooks, Anal. Chem., 842528 (2012) 
23. D. Du, J. Wang, L. Wang, D. Lu, Y. Lin, Anal. Chem., 841380 (2012)

24. L. Wang, D. Lu, J. Wang, D. Du, Z. Zou, H. Wang, J.N. Smith, C. Timchalk, F. Liu, Y. Lin, Biosens. Bioelectron., 262835 (2010)

25. G. Liu, Y.-Y. Lin, J. Wang, H. Wu, C.M. Wai, Y. Lin, Anal. Chem., 797644 (2007)

26. K.S. Lee, T.-H. Kim, M.-C. Shin, W.-Y. Lee, J.-K. Park, Anal. Chim. Acta, 38017 (1999)

27. M. Yan, D. Zang, S. Ge, L. Ge, J. Yu, Biosens. Bioelectron., 38355 (2012)

28. P. Wang, L. Ge, M. Yan, X. Song, S. Ge, J. Yu, Biosens. Bioelectron., 32238 (2011)

29. K.K. Jagadeesan, S. Kumar, G. Sumana, Electrochem. Commun., 2071 (2012)

30. A.C. Siegel, S.T. Phillips, M.D. Dickey, N. Lu, Z. Suo, G.M. Whitesides, Adv. Funct. Mater., 20 $28(2010)$

31. X. Liu, M. Mwangi, X. Li, M. O'Brien, G.M. Whitesides, Lab Chip, 112189 (2011)

32. O.D. Renedo, M.A. Alonso-Lomillo, M.J.A. Martinez, Talanta, 73202 (2007)

33. S.A. Wring, J.P. Hart, L. Bracey, B.J. Birch, Anal. Chim. Acta, 231203 (1990)

34. A. Russo, B.Y. Ahn, J.J. Adams, E.B. Duoss, J.T. Bernhard, J.A. Lewis, Adv. Mater., 233426 (2011)

35. K.A. Mirica, J.G. Weis, J.M. Schnorr, B. Esser, T.M. Swager, Angew. Chem. Int. Ed., 5110740 (2012)

36. A.C. Siegel, S.T. Phillips, B.J. Wiley, G.M. Whitesides, Lab Chip, 92775 (2009)

37. A.D. Mazzeo, W.B. Kalb, L. Chan, M.G. Killian, J.-F. Bloch, B.A. Mazzeo, G.M. Whitesides, Adv. Mater., 242850 (2012)

38. F. Eder, H. Klauk, M. Halik, U. Zschieschang, G.n. Schmid, C. Dehm, Appl. Phys. Lett., 842673 (2004)

39. E. Fortunato, N. Correia, P. Barquinha, L. Pereira, G. Goncalves, R. Martins, IEEE Electron. Device Lett., 29988 (2008)

40. R. Martins, P. Barquinha, L. Pereira, N. Correia, G. Gonçalves, I. Ferreira, E. Fortunato, Appl. Phys. Lett., 93203501 (2008)

41. R. Martins, A. Nathan, R. Barros, L.s. Pereira, P. Barquinha, N. Correia, R. Costa, A. Ahnood, I. Ferreira, E. Fortunato, Adv. Mater., 234491 (2011)

42. $\quad$ S. Yun, S.-D. Jang, G.-Y. Yun, J.-H. Kim, J. Kim, Appl. Phys. Lett., 95104102 (2009)

43. D. Nilsson, T. Kugler, P.-O. Svensson, M. Berggren, Sens. Actuators, B, 86193 (2002)

44. Cost based on ordering 10,000 operational amplifiers from Digi-Key Corp. for $\sim$ US $\$ 0.23$ each. 
45. Z. Weng, Y. Su, D.-W. Wang, F. Li, J. Du, H.-M. Cheng, Adv. Energy Mater., 1917 (2011)

46. M. Hilder, B. Winther-Jensen, N.B. Clark, J. Power Sources, 1941135 (2009)

47. L. Hu, H. Wu, F. La Mantia, Y. Yang, Y. Cui, ACS Nano, 45843 (2010)

48. G. Nyström, A. Razaq, M. Strømme, L. Nyholm, A. Mihranyan, Nano Lett., 93635 (2009)

49. M.C. Barr, J.A. Rowehl, R.R. Lunt, J. Xu, A. Wang, C.M. Boyce, S.G. Im, V. Bulović, K.K. Gleason, Adv. Mater., 233500 (2011)

50. J. Wang, Chem. Rev., 108814 (2007)

51. J.M. Ruano-Lopez, M. Agirregabiria, G. Olabarria, D. Verdoy, D.D. Bang, M. Bu, A. Wolff, A. Voigt, J.A. Dziuban, R. Walczak, J. Berganzo, Lab Chip, 91495 (2009)

52. http://www.anywherescience.com/

53. https://itunes.apple.com/us/app/imultimeter/id420797671? $\mathrm{mt}=8$

54. J.L. Delaney, C.F. Hogan, J. Tian, W. Shen, Anal. Chem., 831300 (2011)

55. L. Ge, J. Yan, X. Song, M. Yan, S. Ge, J. Yu, Biomaterials, 331024 (2011)

56. C.D. Chin, V. Linder, S.K. Sia, Lab Chip, 741 (2007) 